\title{
A vantagem doméstica em plantios homogêneos e floresta nativa em Irati, Paraná
}

\author{
Juliana HANKE¹, Kelly Geronazzo MARTINS'1, Vânia Rossetto MARCELINO2*, \\ Edenilson Luiz PEREIRA FILHO², Leandro TONHATO2 Kátia Cylene LOMBARDI² \\ ${ }^{1}$ Departamento de Engenharia Ambiental, Universidade Estadual do Centro-Oeste, Irati, PR, Brasil. \\ (Orcid: 0000-0003-1519-7531; 0000-0002-0447-4444) \\ 2Departamento de Engenharia Florestal, Universidade Estadual do Centro-Oeste, Irati, PR, Brasil. \\ (Orcid: *; 0000-0002-3136-3651; 0000-0001-7860-681X; 0000-0003-2388-2985) \\ *E-mail: vania@unicentro.br (Orcid: 0000-0001-8511-2324)
}

Recebido em 13/02/2020; Aceito em 28/10/2020; Publicado em 18/11/2020.

\begin{abstract}
RESUMO: O objetivo desse trabalho foi determinar se a respiração microbiana e os atributos pedológicos variam conforme a cobertura florestal e se existe especificidade da micro e macrofauna no processo de decomposição da fitomassa de diferentes áreas florestais. Para tanto foram selecionadas três áreas: um talhão de Pinus taeda, um de Eucalyptus dunnii e um fragmento de Floresta Ombrófila Mista Montana, todas no município de Irati, estado do Paraná. Em cada área foram alocados 288 sacos de serapilheira, nos quais $8 \mathrm{~g}$ de material recém depositado e seco ao ar livre foram confinados. De modo a verificar o efeito da macrofauna, 144 sacos com malha de $1 \mathrm{~mm}$ e 144 sacos de malha de $5 \mathrm{~mm}$ foram distribuídos nos trechos de estudo. Os sacos de decomposição continham fitomassa oriunda do próprio sítio e concomitantemente fitomassa das demais áreas. Os resultados comprovaram que os atributos pedológicos e a respiração microbiana $\left(\mathrm{F}_{2 ; 11}: 13\right.$,2; $\mathrm{p}<0,01)$ oscilam significativamente conforme muda a cobertura vegetal. Após 180 dias de material em campo foi constatado que a macrofauna interfere no processo de decomposição $\left(\mathrm{F}_{53 ; 192}\right.$ : 113,06, $\left.\mathrm{p}<0,01\right)$ e que existe uma elevada afinidade entre a macrofauna de cada sítio e a fitomassa produzida.

Palavras-chave: decomposição; litterbags; respiração basal do solo; Floresta Ombrófila Mista.
\end{abstract}

\section{Home field advantage in cultivated and native forests in Irati, Paraná, Brazil}

\begin{abstract}
The main purpose of this study was determined if microbial respiration and pedological attributes vary according to forest cover and if there is interference of micro and macrofauna in the process of litter decomposition. For the study we selected three areas: a Pinus taeda stand, a Eucalyptus dunnii stand and a Mixed Ombrophillous Forest patch, all sites in Irati, Paraná state. In each area were allocated 288 litterbags, in which $8 \mathrm{~g}$ of newly deposited and air dried material were confined. In order to verify the effect of macrofauna, 144 litterbags of $1 \mathrm{~mm}$ mesh and 144 litterbags of $5 \mathrm{~mm}$ mesh were distributed in the areas. The litterbags contein litter originated from the site itself and concomitantly litter from other areas. The results showed that soil attributes and microbial respiration $\left(\mathrm{F}_{2,11}: 13.2, \mathrm{p}<0.01\right)$ change significantly as the vegetation cover. After 180 days, it was found macrofauna interference in decomposition process $\left(\mathrm{F}_{53}, 192: 113.06, \mathrm{p}<0.01\right)$ and a high affinity between the macrofauna of each site and litterfall.

Keywords: decomposition; litterbags; soil basal respiration; Mixed Ombrophilous Forest.
\end{abstract}

\section{INTRODUÇÃO}

A atual crise de perda de espécies, causada principalmente pela degradação ou corte de florestas tropicais, e que é comparada com a do período Ordoviciano, na qual o planeta chegou a perder cerca de $20 \%$ dos seus taxa, levou à criação de uma série de políticas e esforços, tanto para conservar como para compreender o papel da biodiversidade no planeta e os serviços ambientais ou ecossistêmicos por ela prestados (TOWSEND et al., 2010).

Os serviços ecossistêmicos podem ser divididos em: provedores, de recreação, suporte e reguladores (MILLENNIUM ECOSYSTEM ASSESSMENT - MEA, 2005). Os serviços de suporte fazem referência aos serviços necessários para a produção de todos os outros. São exemplos a ciclagem de nutrientes e a produção primária.

A ciclagem de nutrientes consiste no fluxo e acúmulo dos elementos minerais entre os compartimentos do ecossistema e as transferências entre eles. É esta ciclagem, em especial nos trópicos úmidos, que provê o que talvez seja o mais importante dos serviços ecossistêmicos, que é o de suporte.

Uma das maneiras possíveis de se analisar o ciclo de minerais em florestas é através do estudo da deposição e decomposição da serapilheira. A serapilheira também pode ser denominada folhedo, manta, folhiço, litter ou liteira e constitui todos os tipos de material biogênico em vários estágios de decomposição. É formada principalmente por folhas (fitomassa), além de ramos, caules, flores, frutos, cascas, dejetos e restos animais (MASON, 1980).

A decomposição da liteira é a principal forma de transferência de elementos da vegetação para o solo. Este processo complexo é influenciado por diversos fatores como relevo, vegetação, estágio sucessional, luminosidade, características do solo, disponibilidade de água no ambiente, comunidade de macro e microdecompositores, entre outros (GIEßELMANN et al., 2011). Todavia, tanto a hierarquia como a força de interação entre os fatores acima citados, 
pode ser distinta, tanto entre biomas como entre ecossistemas (FANIN et al., 2019; GARCIA-PALACIOS et al., 2013).

Segundo Gholz et al. (2000), existe uma relação estreita entre a comunidade decompositora e as espécies arbóreas de uma mesma área. Esta especialização caracteriza a teoria da vantagem doméstica (home field advantage). Tal teoria é baseada na premissa de que a micro e a macrofaunas de cada local tem mais afinidade com a liteira produzida in situ e, portanto, decompõe mais rápido do que uma liteira produzida ex situ (AYRES et al., 2006).

Dois fatores são fundamentais para que se confirme a teoria da vantagem doméstica. O primeiro é que a serapilheira deve ser de baixa qualidade, com grande quantidade de material recalcitrante ou compostos tóxicos, para limitar os organismos decompositores, já que uma serapilheira repleta de nutrientes seria altamente palatável para os organismos, sendo consumida sem restrições entre as populações de decompositores (GIEßELMANN et al., 2011).

O outro fator é que a comunidade decompositora deve ser conservadora, pois elevadas alterações nas populações de uma comunidade de decompositores, em um curto período, não possibilitam aferir a especificidade entre a comunidade decompositora e o substrato a ser decomposto, já que esta pode oscilar em um pequeno espaço de tempo, o que geralmente ocorre com a comunidade de microdecompositores (FANIN et al., 2016).

Por ser altamente suscetível a alterações ambientais, a comunidade microbiana do solo pode variar em diferentes tipos de cultivos. Quando a vegetação nativa é substituída por plantios ou consórcios, essa mudança de vegetação altera as características originais do solo, mudando a composição da matéria orgânica, os nutrientes e, por consequência, a comunidade da micro e macrofauna, os quais, entre outros fatores, são parâmetros importantes para a sustentabilidade do solo (HENDRIX et al., 1990).

O solo florestal é um habitat propício ao desenvolvimento microbiano e a participação da biota do solo no funcionamento e sustentabilidade dos ecossistemas é bem reconhecida (FANIN et al., 2016). Os processos microbianos são uma parte integral da qualidade do solo e a atividade dos microrganismos presentes pode servir para a compreensão da estabilidade dentro do processo (GIACOMETTI et al., 2013).

Os microrganismos constituintes da microbiota nos solos são muito sensíveis e podem ser influenciados por fatores tanto bióticos como abióticos. Desta forma, alguns parâmetros referentes à atividade dos microrganismos no solo podem ser utilizados como bioindicadores para avaliação do estado de equilíbrio ou desequilíbrio de ecossistemas florestais (ZILLI et al., 2003; MOREIRA; SIQUEIRA, 2002).

Devido ao clima do município de Irati, as espécies arbóreas comerciais mais plantadas para produção de madeira na região são Pinus taeda e Eucalyptus dunnii. As florestas homogêneas formadas ora pelos plantios de Pinus taeda, ora pelos plantios de Eucalyptus dunnii, entre outras espécies arbóreas comerciais, substituem o ecossistema florestal original da região, denominado Floresta Ombrófila Mista e, depois dos ecossistemas agrossilviculturais, as florestas homogêneas são o que mais se assemelha a florestas nativas em termos de estrutura (BERNARDINO; GARCIA, 2009). Neste contexto, o presente estudo buscou testar as seguintes hipóteses: solos com diferentes coberturas florestais irão apresentar atributos pedológicos e respiração microbiana distintos entre si; a comunidade de macrodecompositores desenvolve um papel significativo na mineralização da matéria orgânica; devido à elevada especialização entre decompositores e substrato de decomposição, áreas com diferentes coberturas florestais, em especial monoculturas, irão apresentar dinâmicas de decomposição mais rápidas em seu ambiente do que fora dele, conforme estabelecido pela teoria da vantagem doméstica.

Assim, os objetivos da pesquisa são: aferir a respiração basal do solo nos trechos amostrados; contrastar o material remanescente nos litterbags das diferentes coletas em função do tamanho da abertura da malha; avaliar a hipótese da vantagem doméstica.

\section{MATERIAL E MÉTODOS}

\section{1. Áreas de estudo}

O estudo foi realizado em uma área no Campus da UNICENTRO, localizado no município de Irati (PR), e em duas áreas no entorno da universidade, uma de plantio de Eucalyptus dunnii (Figura 1A) e outra de Floresta Ombrófila Mista Montana (Figura 1C). A área que está dentro do Campus refere-se a um talhão de 1,65 ha de Pinus taeda (Figura 1B), plantado em 2003. As mudas de P. taeda foram fornecidas pela empresa Klabin e nessa plantação ocorreu aplicação de formicida (aproximadamente 2,0 $\mathrm{kg} \mathrm{ha}^{-1}$ ) também em 2003. O plantio de E. dunnii (Figura 1A) tem aproximadamente oito anos e não ocorreram aplicações de formicida ou qualquer outro defensivo no trecho que tem 1 ha. Finalmente, o trecho coberto por Floresta Ombrófila Mista (Figura 1C), ou Floresta com Araucária, tem 3 ha e está em estágio sucessional inicial, com um sub-bosque em formação. Os dois últimos trechos localizam-se ao lado do campus.

As variáveis climáticas temperatura média do ar e precipitação total do período de abril a setembro de 2013 (tempo do experimento) foram cedidas pelo Sistema Meteorológico do Paraná - SIMEPAR, e são relativas ao município de Fernandes Pinheiro, vizinho cuja distância central de Irati é de cerca de $11 \mathrm{~km}$.

No período em que o experimento foi realizado, a menor temperatura aconteceu no mês de julho, a qual foi de $-2,7^{\circ} \mathrm{C}$. A temperatura máxima foi de $30,4^{\circ} \mathrm{C}$, em setembro. $\mathrm{O}$ maior índice de pluviosidade foi de $113,6 \mathrm{~mm}$, em junho, o que demostra um ano atípico, já que historicamente esse período é caracterizado pela baixa pluviosidade. As temperaturas médias e a precipitação total ao longo dos seis meses podem ser visualizadas na Figura 2.

\subsection{Fitossociologia do fragmento de floresta nativa}

Foram instaladas oito parcelas de $10 \times 10 \mathrm{~m}$ no fragmento de Floresta Ombrófila Mista. De todos os indivíduos em que foi possível alcançar material botânico, fez-se a coleta e posterior confecção de exsicatas, as quais estão depositadas como testemunho no Herbário da Universidade Estadual do Centro-Oeste (HUCO). Foi medido o perímetro a 1,3m do solo de todos os indivíduos amostrados. Entraram na amostragem os de porte arbóreo com no mínimo $10 \mathrm{~cm}$ de circunferência a $1,3 \mathrm{~m}$ do solo.

\subsection{Análise físico-química do solo}

Foram coletadas amostras de solos nas três áreas de estudo (plantio de Pinus, plantio de Eucalipto e Floresta 
Ombrófila Mista). Cada área foi dividida em quatro parcelas, com $100 \mathrm{~m}^{2}$ cada. Dentro destas quatro parcelas foram coletadas, com um anel de $5 \mathrm{~cm}$ de profundidade, amostras de solo em cinco pontos aleatórios, obtendo-se assim uma amostra composta de cada parcela.

As amostras foram analisadas pelo Laboratório de
Análises de Rotina de Solos da UNICENTRO, Campus de Irati, Departamento de Engenharia Florestal. A metodologia utilizada para a obtenção da matéria orgânica foi digestão úmida. $\mathrm{O} \mathrm{P}$ e o $\mathrm{K}$ foram extraídos com solução de Mehlich1, o pH em $\mathrm{CaCl}_{2}$ 1:2:5 e Ca, $\mathrm{Mg}$ e $\mathrm{Al}$ trocáveis extraídos com $\mathrm{KCl} 1 \mathrm{~mol} \mathrm{~L}-1$.
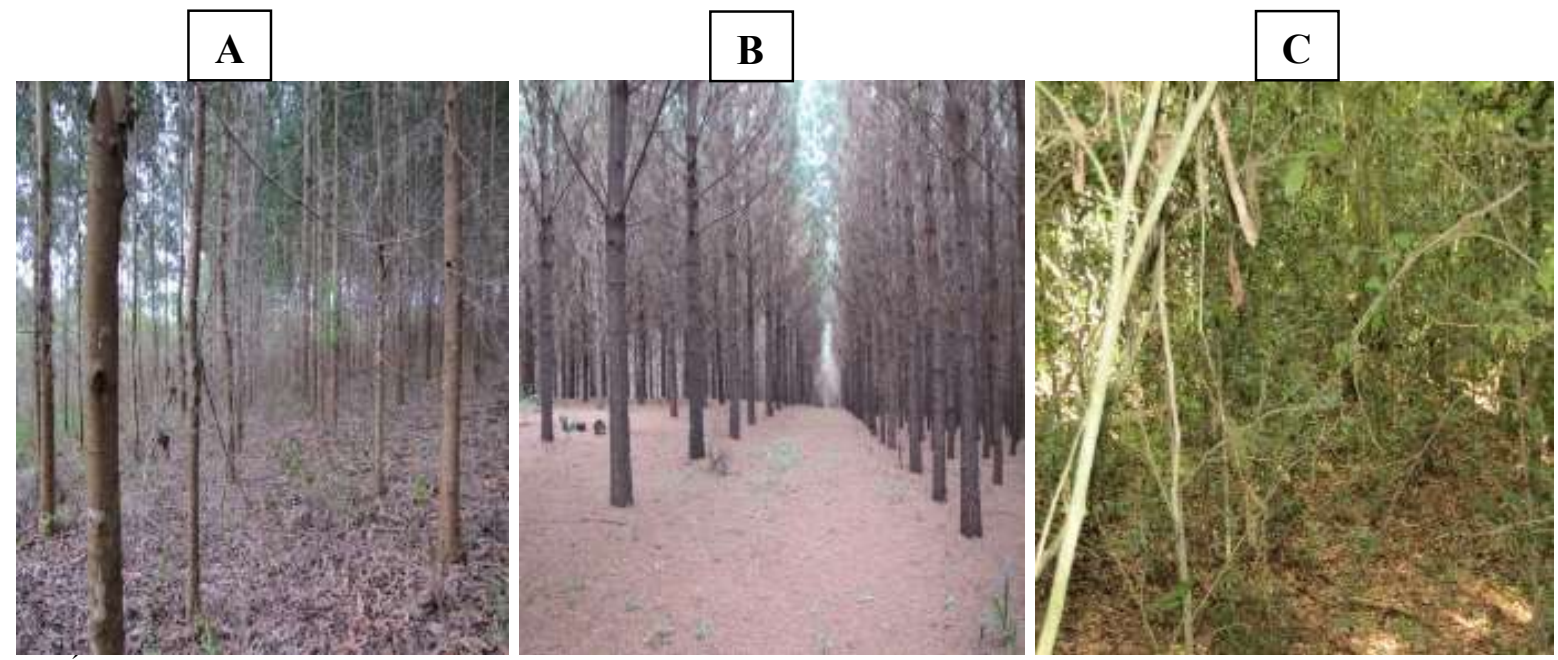

Figura 1. Áreas de (A) Eucalyptus dunnii, (B) Pinus taeda e (C) Floresta Ombrófila Mista em Irati, Paraná. Autor: Juliana Hanke.

Figure 1. (A) Eucalyptus dunnii, (B) Pinus taeda and (C) Mixed Ombrophilous Forest areas in Irati, Paraná, Brazil. Author: Juliana Hanke.

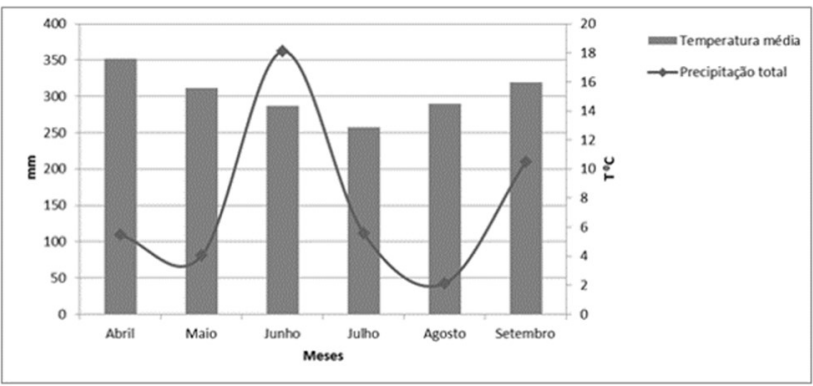

Figura 2. Gráfico de temperaturas médias e precipitações totais entre os meses de abril e setembro de 2013. Fonte de dados: Sistema Meteorológico do Paraná - SIMEPAR.

Figure 2. Graphic of average temperatures and total precipitation along April and September 2013. Data source: Paraná Weather System - SIMEPAR.

Para testar a hipótese de que solos do mesmo tipo, porém com diferentes coberturas florestais irão apresentar atributos pedológicos distintos entre si, foi realizada uma Análise de Componentes Principais com os seguintes atributos: alumínio, argila, silte, areia fina, areia grossa, matéria orgânica, fósforo, $\mathrm{pH}$ e potássio.

A análise de componentes principais (ACP) é uma análise de ordenação cujo objetivo é reduzir a complexidade dos dados, representando-os num sistema de novas variáveis ou novas dimensões (eixos). Mediante a correlação das unidades amostrais do estudo com os eixos das componentes principias é possível visualizar como as unidades amostrais (parcelas) ficaram ordenadas. A ACP foi calculada a partir da matriz de correlação para evitar distorções entre variáveis de diferentes unidades. O número de componentes principais (eixos) foi determinado previamente mediante o critério de Kaiser (McCUNE; GRACE, 2002).

\subsection{Decomposição da serapilheira}

No total foram alocados 288 sacos de liteira (litterbags), contendo $8 \mathrm{~g}$ de serapilheira recém depositada e seca ao ar livre. Destes 288 sacos, 144 eram de malha fechada (abertura de $1 \mathrm{~mm}$ de diâmetro) e 144 eram de malha aberta $(5 \mathrm{~mm})$. Os sacos de malha fechada foram utilizados com objetivo de impedir a entrada da macro e meso fauna do solo, enquanto os de malha aberta permitiram a entrada destes decompositores (Tabela 1).

Tabela 1. Esquema de exclusão/inclusão dos organismos decompositores presentes na serapilheira, conforme o tamanho das malhas utilizadas na confecção dos litterbags.

Table 1. Scheme of exclusion/inclusion of decomposing organisms present in the litter, according to the size of the meshes used to make the litterbags.

\begin{tabular}{lll}
\hline Malha & Exclusão & Inclusão \\
\hline Aberta & Macrofauna $(>4 \mathrm{~mm})$ & Macrofauna $(<5 \mathrm{~mm})$ \\
$(5 \mathrm{~mm})$ & & Microfauna \\
& & Mesofauna \\
& & Microflora \\
\hline Fechada & Macrofauna & Microfauna $(<1 \mathrm{~mm})$ \\
$(1 \mathrm{~mm})$ & Mesofauna & Microflora \\
& Microfauna $(>4 \mathrm{~mm})$ & \\
\hline
\end{tabular}

Especificamente 48 sacos de malha fechada e 48 de malha aberta foram alocados em cada um dos ecossistemas; estes 48 sacos (tanto para a malha aberta como a fechada) são formados por 3 grupos de 16 sacos com 8 gramas da serapilheira recém depositada de cada um dos ecossistemas pertinentes a este estudo (Figura 3). Assim, cada ecossistema recebeu sacos contendo serapilheira dele e dos outros dois ecossistemas, de modo a se verificar o possível efeito da vantagem doméstica.

Foram efetuadas quatro coletas: a primeira após 15 dias, a segunda após 45 dias, e as duas últimas após 75 e 180 dias de material em campo. Após as coletas o material foi seco até peso constante e sua massa anotada em planilhas para posterior análise da porcentagem de perda de peso (peso inicial $=100 \%$, peso da coleta $=\mathrm{x}$ ).

$\mathrm{O}$ experimento seguiu um delineamento hierarquizado, 
com quatro repetições por ecossistema. As porcentagens de perda de peso foram consideradas as variáveis dependentes e o ecossistema o fator; dentro deste fator (ecossistemas) ficaram aninhados os fatores: tipo de malha (aberta e fechada) e tipo de fitomassa (Eucalyptus dunnii, Floresta Ombrófila
Mista e Pinus taeda). A checagem prévia da homogeneidade das variâncias foi efetuada pelo teste de Bartlett (ZAR, 1999). A comparação entre as médias foi efetuada pelo teste de Tukey a $5 \%$.
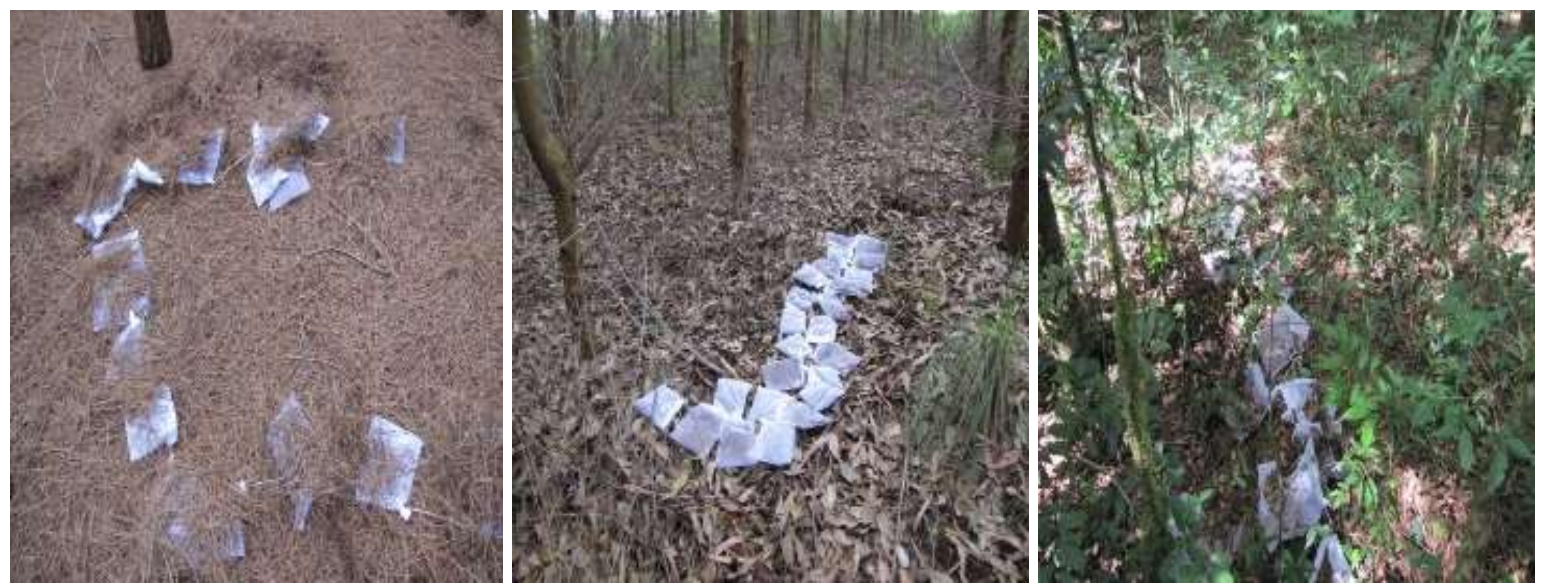

Figura 3. Litterbags dispostos nas áreas de Pinus taeda, Eucalyptus dunnii e Floresta Ombrófila Mista. Autor: Juliana Hanke.

Figure 3. Litterbags placed in the Pinus taeda, Eucalpptus dunnii and Mixed Ombrophilous Forest areas. Author: Juliana Hanke.

\subsection{Determinação da respiração do solo}

Para a determinação da respiração microbiana utilizou-se o método de Jenkinson; Powlson (1976), pelo qual a respiração é avaliada após a incubação de $30 \mathrm{~g}$ de solo úmido juntamente com tubos de ensaio contendo água deionizada (para manutenção da umidade da amostra) e $\mathrm{NaOH}(0,5 \mathrm{~mol}$ $\left.\mathrm{L}^{-1}\right)$, por um período de sete dias em temperatura ambiente. Neste método, o $\mathrm{CO}_{2}$ produzido pela respiração dos microrganismos reage com o hidróxido de sódio $(\mathrm{NaOH})$, e o excedente é titulado com $\mathrm{HCl}\left(0,5 \mathrm{~mol} \mathrm{~L}^{-1}\right)$. Para a titulação utilizou-se uma alíquota de $10 \mathrm{~mL}$ de $\mathrm{NaOH}$ com $1 \mathrm{~mL}$ de $\mathrm{BaCl}_{2}(50 \%)$ e três gotas de fenolftaleína (1\%). O cálculo da taxa de respiração (Equação 1) foi efetuado conforme Jenkinson; Powlson (1976).

As amostras foram coletadas da mesma forma que para as análises químicas e físicas de solo. As coletas foram efetuadas em junho de 2013 e no mesmo dia foram processadas e incubadas por uma semana para então serem tituladas. Todos os procedimentos analíticos foram realizados no Laboratório de Química do Departamento de Engenharia Ambiental da UNICENTRO, Campus de Irati.

$$
R \mathrm{BS}=\frac{\left(\frac{\left(\mathrm{v}_{\mathrm{a}}-\mathrm{V}_{\mathrm{b}}\right) \times \mathrm{M} \times 6 \times 10}{\mathrm{Ps}}\right)}{\mathrm{T}}
$$

em que: $\mathrm{RBS}=$ Carbono oriundo da respiração do solo, em mg de $\mathrm{C}-\mathrm{CO}_{2} \mathrm{Kg}^{-1}$ Solo hora ${ }^{-1} ; \mathrm{V}_{\mathrm{b}}=$ Volume de ácido clorídrico gasto na titulação da solução controle; $\mathrm{V}_{\mathrm{a}}=$ Volume gasto na titulação da amostra; M = Molaridade exata do HCL; Ps (g) = Massa de solo seco; $\mathrm{T}=$ Tempo de incubação das amostras em horas.

Para testar a hipótese de que a respiração microbiana (RM) difere em solos com diferentes coberturas, os dados de RM foram submetidos à análise de variância com um fator (área de estudo); a variável dependente foi a respiração microbiana. O delineamento foi inteiramente casualizado. As médias foram calculadas a partir de quatro repetições e comparadas pelo teste de Tukey. A homogeneidade de variância foi verificada previamente pelo teste de Bartlett. A significância para todos os testes foi de 5\% (ZAR, 1999).

\section{RESULTADOS}

\subsection{Fitossociologia}

Foram registrados 244 indivíduos, divididos em 36 espécies (Tabela 2) e 17 famílias. As famílias com maior número de espécies foram: Myrtaceae e Salicaceae (6 espécies cada); e Euphorbiaceae (4 espécies). A Frequência Acumulada de Espécies arbóreas na amostragem é apresentada na Figura 4. Percebe-se, pela curva em estabilização da frequência acumulada, que a amostragem foi suficiente para incluir a grande maioria das espécies arbóreas. O gráfico de frequência acumulada em função da área é uma forma de determinar a intensidade amostral (MAGURRAN, 2011).

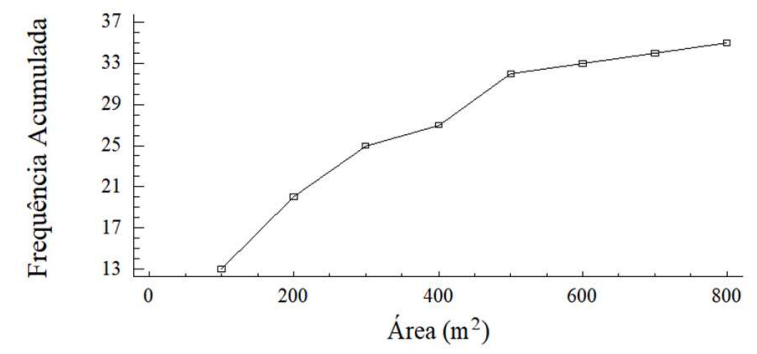

Figura 4. Frequência Acumulada (F.A.) de espécies arbóreas, por área amostrada no fragmento de Floresta Ombrófila Mista.

Figure 4. F.A = Cumulative Frequency (F.A.) of tree species, by sampled area in the Mixed Ombrophilous Forest fragment.

Dos indivíduos dos quais foram feitas coletas botânicas, as exsicatas estão depositadas no herbário HUCO sob os números 7074 a 7228 e 7231 a 7280.

O fragmento pode ser considerado em estágio inicial de regeneração, conforme a classificação de Castella; Britez (2004). A Figura 5 mostra um diagrama de perfil do fragmento para auxiliar a visualizar seus estratos e seu estado de conservação.

\subsection{Composição químico-física do solo}

De forma geral o Cálcio, o Alumínio, a Matéria Orgânica 
(M.O.) e o $\mathrm{V} \%$ foram as variáveis que diferiram de forma mais acentuada entre as áreas (Tabela 3). Esses atributos apresentaram maiores valores no trecho de floresta nativa, resultado que está relacionado à matéria orgânica. Provavelmente os trechos de floresta nativa depositam mais serapilheira e, portanto, acumulam maior quantidade de matéria orgânica no solo (AYRES et al., 2006).

A matéria orgânica, por sua vez, aumenta o $\mathrm{V} \%$ e por consequência os teores de $\mathrm{Ca}$ no solo. Outra propriedade importante da M.O. é que ela pode vir a imobilizar o alumínio
(CARVALHO, 1999).

A análise de componentes principais (Figura 6) ordenou os trechos amostrados em três grupos distintos (área de Eucalyptus dunnii, Floresta Ombrófila Mista e Pinus taeda). Esses resultados demonstram que a partir dos 12 atributos analisados, as áreas diferem significativamente entre si. As variáveis mais importantes para a ordenação foram cálcio, alumínio, matéria orgânica e V\% (atributo de fertilidade). Os mesmos atributos foram visualizados na Tabela 3 com os valores mais discrepantes entre os trechos.

Tabela 2. Espécies encontradas no fragmento de Floresta Ombrófila Mista. $\mathrm{N}=$ número de indivíduos registrados por espécie. IVI = Índice de Valor de Importância. IVC = Índice de Valor de Cobertura.

Table 2. Species recorded in the Mixed Ombrophilous Forest fragment. $\mathrm{N}=$ number of registered individuals per species. IVI = Importance Value Index. IVC = Coverage Value Index.

\begin{tabular}{|c|c|c|c|}
\hline Espécie & $\mathrm{N}$ & IVI & IVC \\
\hline Allophylus edulis (A. St.-Hil., A. Juss. \& Cambess.) Hieron. ex Niederl. & 1 & 1,70 & 0,77 \\
\hline Banara tomentosa Clos & 1 & 1,39 & 0,45 \\
\hline Campomanesia xanthocarpa Mart. ex O. Berg & 4 & 4,99 & 2,18 \\
\hline Casearia decandra Jacq. & 9 & 9,17 & 5,43 \\
\hline Casearia lasiophylla Eichler & 7 & 9,18 & 4,51 \\
\hline Casearia obliqua Spreng. & 24 & 31,70 & 24,22 \\
\hline Casearia silvestris $\mathrm{Sw}$ & 15 & 17,02 & 10,47 \\
\hline Celtis iguanaea (Jacq.) Sarg. & 1 & 1,69 & 0,76 \\
\hline Cinnamodendron dinisii Schwacke & 29 & 35,45 & 28,91 \\
\hline Cupania vernalis Cambess. & 2 & 2,76 & 0,89 \\
\hline Curitiba prismatica (D. Legrand) Salywon \& L.R. Landrum & 9 & 9,27 & 5,53 \\
\hline Diatenopteryx sorbifolia Radlk. & 7 & 11,38 & 8,57 \\
\hline Erythroxylum deciduum A. St.-Hil. & 1 & 1,90 & 0,97 \\
\hline Eugenia uniflora $\mathrm{L}$. & 23 & 26,54 & 19,06 \\
\hline Eugenia uruguayensis Cambess. & 13 & 13,06 & 6,52 \\
\hline Gymnanthes brasiliensis (Spreng.) Müll.Arg. & 22 & 16,95 & 11,34 \\
\hline Gymnanthes concolor (Spreng.) Müll. Arg. & 10 & 7,49 & 4,69 \\
\hline Gymnanthes klotzschiana Müll.Arg. & 26 & 23,74 & 16,26 \\
\hline Ilex paraguariensis A. St.-Hil. & 2 & 2,83 & 0,96 \\
\hline Lafoensia pacari A. St.-Hil. & 7 & 14,25 & 10,51 \\
\hline Lithraea brasiliensis Marchand & 1 & 2,47 & 1,53 \\
\hline Luehea divaricata Mart. & 2 & 4,20 & 2,33 \\
\hline Machaerium brasiliense Vogel & 2 & 4,18 & 3,24 \\
\hline Machaerium stipitatum (DC.) Vogel & 1 & 1,68 & 0,75 \\
\hline Maytenus aquifolia Mart. & 1 & 1,38 & 0,45 \\
\hline Myrcia splendens (Sw.) DC. & 1 & 1,53 & 0,60 \\
\hline Myrciaria floribunda (H. West ex Willd.) O. Berg & 1 & 1,41 & 0,48 \\
\hline Não identificada & 5 & 5,76 & 2,96 \\
\hline Nectandra grandiflora Nees \& Mart. ex Nees & 1 & 1,49 & 0,56 \\
\hline Ocotea diospyrifolia (Meisn.) Mez & 4 & 4,64 & 1,84 \\
\hline Parapiptadenia rigida (Benth.) Brenan & 6 & 17,02 & 16,09 \\
\hline Solanum sanctaecatharinae Dunal & 1 & 1,43 & 0,50 \\
\hline Strychnos brasiliensis (Spreng.) Mart. & 1 & 1,38 & 0,45 \\
\hline Syagrus romanzoffiana (Cham.) Glassman & 1 & 1,67 & 0,74 \\
\hline Xylosma pseudosalzmanii Sleumer & 1 & 1,88 & 0,94 \\
\hline Zanthoxylum kleinii (R.S. Cowan) P.G. Waterman & 2 & 5,40 & 3,54 \\
\hline Total geral & 244 & & \\
\hline
\end{tabular}

\subsection{Respiração microbiana}

Os valores de respiração do solo encontrados foram: $85,75 \mathrm{mg} . \mathrm{C}-\mathrm{CO}_{2} \cdot \mathrm{h}^{-1} \cdot 30 \mathrm{gss}^{-1}, 77,8385,75 \mathrm{mg} \cdot \mathrm{C}-\mathrm{CO}_{2} \cdot \mathrm{h}^{-1} \cdot 30 \mathrm{gss}$ 1 e $63,2185,75 \mathrm{mg} . \mathrm{C}-\mathrm{CO}_{2} \cdot \mathrm{h}^{-1} \cdot 30 \mathrm{gss}^{-1}$ para FOM (Floresta Ombrófila Mista), Plantio de Eucalyptus dunnii e Pinus taeda, respectivamente. Estes valores estão em consonância com os verificados na literatura para florestas nativas e plantios de Pinus taeda e Eucalyptus dunnii (ASSIS JÚNIOR et al., 2003).

A comparação estatística das médias demonstrou que a respiração microbiana do solo oscilou significativamente conforme a cobertura florestal $\left(\mathrm{F}_{2 ; 11}: 13,2 ; \mathrm{p}<0,01\right)$, sendo que o plantio de E. dunnii apresentou a média estatisticamente igual à da região de Floresta Ombrófila Mista e o Plantio de P. taeda a média mais distante (Figura 7).

\subsection{Decomposição da serapilheira}

De forma geral os resultados apresentados descrevem as fases inicial e intermediária da decomposição da serapilheira (Figura 8), em que pelo menos $50 \%$ do material é decomposto (MASON et al., 2011), com exceção para a decomposição das acículas de Pinus na área de Eucalipto, na qual após 180 dias de material em campo, o peso remanescente ainda estava em torno de $75 \%$.

$\mathrm{Na}$ área de Floresta Ombrófila Mista, alguns litterbags que continham acículas de Pinus foram extraviados, provavelmente por carreamento pela fauna local. Por esse motivo não existem resultados de porcentagem de material remanescente referente ao Pinus na área de Floresta Ombrófila Mista (Figura 9). Tal especulação sobre o 
carreamento dos litterbags se deve ao fato de alguns sacos terem sido encontrados na entrada e no interior de uma toca de tatu.

Tabela 3. Atributos pedológicos: médias e desvio padrão referentes às áreas de Eucalyptus dunnii, Floresta Ombrófila Mista (FOM) e Pinus taeda no município de Irati, PR.

Table 3. Pedological attributes: means and standard deviation for Eucalyptus dunnii, Mixed Ombrophilous Forest and Pinus taeda areas in Irati, Paraná, Brazil.

\begin{tabular}{llll}
\hline Dados & Eucalipto & FOM & Pinus \\
\hline $\mathrm{pH}$ & $4,2 \pm 0,12$ & $4,48 \pm 0,25$ & $4,2 \pm 0,08$ \\
$\mathrm{~K}\left(\mathrm{cmol}_{\mathrm{c}} / \mathrm{dm}^{3}\right)$ & $0,52 \pm 0,02$ & $0,61 \pm 0,02$ & $0,51 \pm 0,08$ \\
$\mathrm{Ca}\left(\mathrm{cmol}_{\mathrm{c}} / \mathrm{dm}^{3}\right)$ & $3,68 \pm 0,68$ & $6 \pm 1,41$ & $3,05 \pm 0,48$ \\
$\mathrm{Mg}\left(\mathrm{cmol}_{\mathrm{c}} / \mathrm{dm}^{3}\right)$ & $2,75 \pm 1,10$ & $3,3 \pm 1,00$ & $2,58 \pm 0,85$ \\
$\mathrm{Ca}+\mathrm{Mg}\left(\mathrm{cmol}_{\mathrm{c}} / \mathrm{dm}^{3}\right)$ & $6,43 \pm 1,29$ & $9,3 \pm 2,17$ & $5,63 \pm 0,44$ \\
$\mathrm{Al}\left(\mathrm{cmol}_{\mathrm{c}} / \mathrm{dm}^{3}\right)$ & $4,45 \pm 0,79$ & $1,16 \pm 0,94$ & $3,65 \pm 1,85$ \\
$\mathrm{M} . \mathrm{O}\left(\mathrm{g} / \mathrm{dm}^{3}\right)$ & $45,2 \pm 3,51$ & $66,32 \pm 0,94$ & $36,35 \pm 4,18$ \\
$\mathrm{P}\left(\mathrm{mg} / \mathrm{dm}^{3}\right)$ & $30,45 \pm 15,75$ & $10,68 \pm 1,00$ & $8,47 \pm 2,26$ \\
$\mathrm{~V} \%(\mathrm{~g} / 100 \mathrm{~g})$ & $37,8 \pm 5,69$ & $52,2 \pm 11,81$ & $36,1 \pm 3,41$ \\
Areia Grossa $(\mathrm{g} / 100 \mathrm{~g})$ & $2,48 \pm 0,89$ & $12,07 \pm 6,77$ & $3,35 \pm 0,83$ \\
Areia Fina $(\mathrm{g} / 100 \mathrm{~g})$ & $2,35 \pm 0,38$ & $4,85 \pm 0,44$ & $2,73 \pm 0,57$ \\
Silte $(\mathrm{g} / 100 \mathrm{~g})$ & $38,43 \pm 1,20$ & $41,08 \pm 1,77$ & $42,93 \pm 3,97$ \\
Argila $(\mathrm{g} / 100 \mathrm{~g})$ & $56,75 \pm 1,92$ & $42 \pm 7,25$ & $51 \pm 3,83$ \\
\hline
\end{tabular}

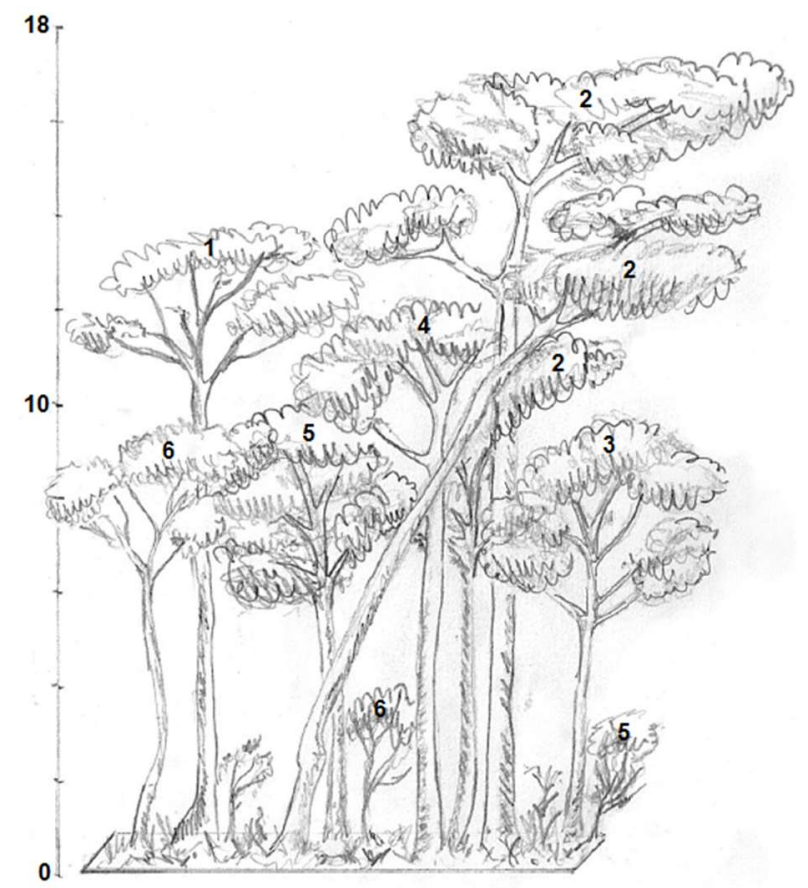

Figura 5. Diagrama de perfil do fragmento de Floresta Ombrófila Mista. 1 - Casearia decandra Jacq. 2 - Casearia obliqua Spreng. 3 Casearia sylvestris Swartz. 4 - Cinnamodendron dinisii Schwanke. 5 Cupania vernalis Cambess. 6 - Diatenopteryx sorbifolia Radlk. Autor: Leandro Tonhato.

Figure 5. Profile diagram of the Mixed Ombrophilous Forest patch. 1 - Casearia decandra Jacq. 2 - Casearia obliqua Spreng. 3 Casearia sylvestris Swartz. 4 - Cinnamodendron dinisii Schwanke. 5 Cupania vernalis Cambess. 6 - Diatenopteryx sorbifolia Radlk. Author: Leandro Tonhato.

\section{DISCUSSÃO}

$\mathrm{Na}$ análise de ordenação, apenas os dois primeiros eixos, ou componentes principais, apresentam autovalores maiores que 1 , e explicam em conjunto cerca de $78,5 \%$ da variação dos dados. Tal porcentagem de explicação é considerada elevada, especialmente quando comparada a resultados de ordenações para dados de experimentos em campo (McCUNE; GRACE, 2002).

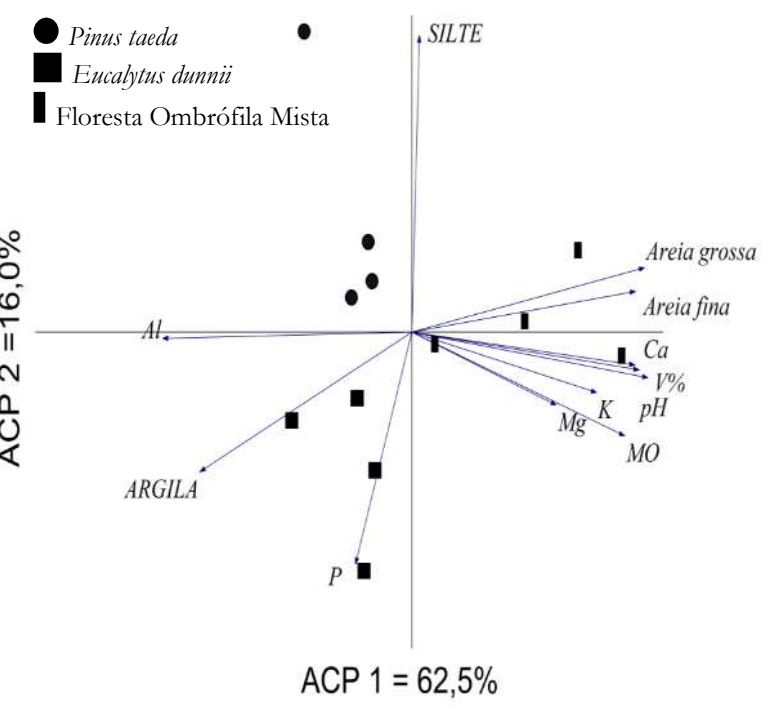

Figura 6. Análise de componentes principais da matriz de atributos pedológicos em três áreas de diferentes coberturas florestais no município de Irati, PR.

Figure 6. Principal component analysis of the pedological attributes matrix in three areas of different forest cover in the municipality of Irati, Paraná, Brazil.

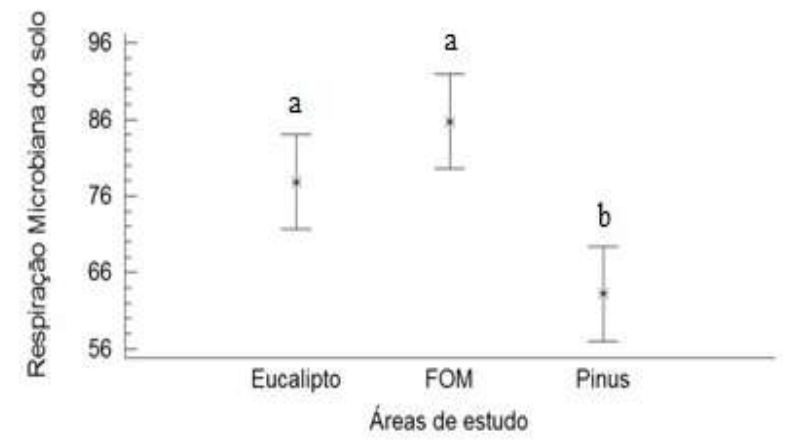

Figura 7. Médias e diferença média significativa do teste de Tukey a $5 \%$ de significância dos valores de respiração microbiana do solo (mg.C-CO $\left.{ }_{2} \cdot \mathrm{h}^{-1} \cdot 30 \mathrm{gss}^{-1}\right)$ em três áreas com diferentes coberturas florestais no município de Irati, PR. FOM = Floresta Ombrófila Mista. Médias com a mesma letra não diferem estatisticamente.

Figure 7. Means and significant mean difference of Tukey test at 5\% significance of soil microbial respiration values (mg.C-CO2.h $1.30 \mathrm{gss}^{-1}$ ) in three areas with different forest cover in the municipality of Irati, Paraná, Brazil. FOM = Mixed Ombrophilous Forest. Means with the same letter do not differ statistically.

Hooper et al. (2004) e Cardoso et al. (2012) já demonstraram que os atributos pedológicos quando submetidos a uma análise de componentes principais expressam uma boa ordenação dos sítios de estudo, como a que foi verificada no presente trabalho. É possível atribuir tal resultado à elevada correlação entre as variáveis.

O resultado da respiração microbiana confirma a hipótese do trabalho de que os processos microbianos são uma parte integral da qualidade do solo e que a atividade destes microrganismos pode servir como indicador biológico para a compreensão da estabilidade e produtividade dentro de um ecossistema. Os microrganismos são muito sensíveis e podem ser influenciados pelos fatores bióticos, como a cobertura florestal, que irá determinar a qualidade da serapilheira, que por sua vez interfere na comunidade 
decompositora e, portanto, nos microrganismos e na respiração microbiana (GIEßELMANN et al., 2011). Fatores abióticos, como temperatura, pluviosidade e $\mathrm{pH}$ do solo também interferem no processo (VARGAS; SCHOLLES, 2000; SMYTH et al., 2011).
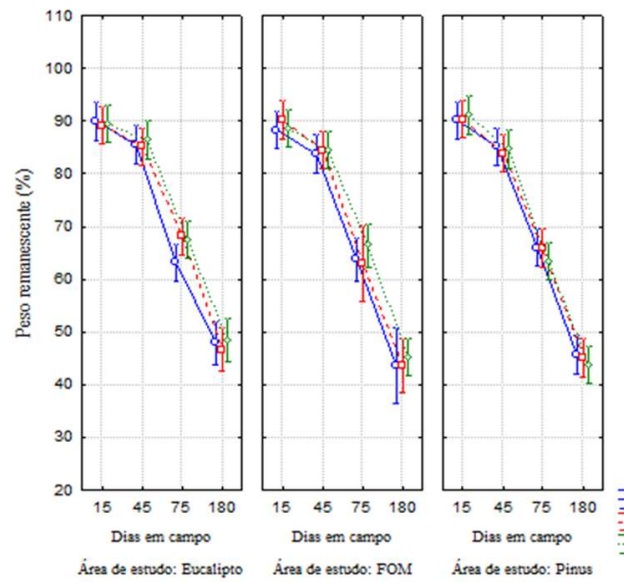

Folhas de FOM Área de estudo: Pinus

Figura 8. Perda de peso das amostras em malha de $1 \mathrm{~mm}$ de abertura, durante os períodos de 15, 45, 75 e 180 dias nas áreas (Eucalyptus dunnii, Floresta Ombrófila Mista e Pinus taeda). Os valores representam as médias e as barras representam as diferenças médias significativas para o teste de Tukey a 5\% de significância.

Figure 8 . Weight loss of samples in $1 \mathrm{~mm}$ mesh during the periods of 15, 45, 75 and 180 days in the areas (Eucalyptus dunnii, Mixed Ombrophilous Forest and Pinus taeda). The values represent the means and the bars represent the significant mean differences for the Tukey test at $5 \%$ significance.
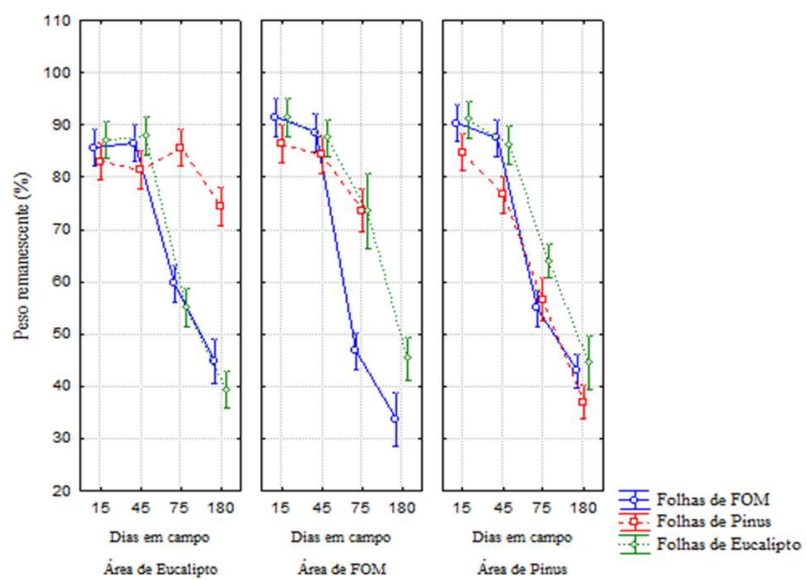

Figura 9. Perda de peso das amostras em malha de $5 \mathrm{~mm}$ de abertura durante os períodos de 15, 45, 75 e 180 dias nas áreas (Eucalyptus dunnii, Floresta Ombrófila Mista e Pinus taeda). Os valores representam as médias e as barras representam as diferenças médias significativas para o teste de Tukey a 5\% de significância.

Figure 9. Weight loss of samples in $5 \mathrm{~mm}$ mesh during the periods of 15, 45, 75 and 180 days in the areas (Eucalyptus dunnii, Mixed Ombrophilous Forest and Pinus taeda). The values represent the means and the bars represent the significant mean differences for the Tukey test at $5 \%$ significance.

No período entre 15 e 45 dias o peso dos litterbags de malha $5 \mathrm{~mm}$ que se encontravam na área de Eucalipto contendo serapilheira de E. dunnii, P. taeda e Floresta Ombrófila Mista não tiveram decréscimo. Provavelmente por conta das condições climáticas durante o período de coleta (temperatura média de $17,15^{\circ} \mathrm{C}$ ). A decomposição no período inicial é reconhecidamente mais rápida; neste estudo deve ter sido retardada devido à baixa temperatura do

período. Heneghan et al. (1998) encontraram o mesmo resultado em um estudo feito em áreas tropicais e temperadas. É bem reconhecido que as condições climáticas influenciam a macro e a mesofauna do solo; nesse sentido, a decomposição após 15 dias foi subestimada devido às condições climáticas desfavoráveis.

A comunidade de macrodecompositores interferiu significativamente na decomposição da serapilheira, pois a dinâmica do peso remanescente durante os 180 dias do material em campo foi distinta entre as diferentes aberturas da malha (Figuras 6 e 7). Observou-se que a perda de peso foi maior nos litterbags que apresentavam malha de abertura igual a $5 \mathrm{~mm}$. Este tamanho de abertura permite o acesso da comunidade de macrodecompositores à serapilheira, diferente da malha de $1 \mathrm{~mm}$. Nos trópicos úmidos a influência da macrofauna na decomposição é bem documentada (LAVELLE et al., 1993; GHOLZ et al., 2000; GUZMÁN; SÁNCHEZ, 2003); Schmidt et al. (2008) e Gießelmann et al. (2010; 2011) também encontraram efeito significativo da comunidade de decompositores na decomposição da serapilheira em trechos de Floresta Atlântica do sul do Brasil, usando a mesma metodologia do presente estudo.

Comparativamente, a serapilheira decompôs mais rápido em seu local de origem somente no ensaio com malha de abertura de $5 \mathrm{~mm}$ (Figura 7), confirmando assim a hipótese da vantagem doméstica (home field advantage), fato que não foi evidenciado no experimento que excluiu os macrodecompositores (malha de $1 \mathrm{~mm}$ ). Entre os poucos trabalhos que testam a vantagem doméstica em ecossistemas tropicais, apenas Gießelmann et al. (2011) conseguiram comprovar parcialmente esta teoria. Os resultados descrevem que existe afinidade entre a comunidade de macrodecompositores com a comunidade arbórea na qual ela está inserida, enquanto os microdecompositores, ainda que variem significativamente entre as áreas, conforme comprovado neste trabalho pelos dados de respiração do solo, não apresentaram a mesma afinidade que a comunidade de macrodecompositores. Possivelmente a explicação para este fato seja a elevada flexibilidade dos microrganismos para se adaptarem em diferentes substratos, trocando a estrutura de sua comunidade em curtos períodos de tempo (OSTERTAG et al., 2008; GIEßELMANN et al., 2011).

Os litterbags que continham serapilheira da Floresta Ombrófila Mista tiveram uma perda de peso uniforme nas três áreas de estudo, diferente das outras espécies, que tiveram uma decomposição mais lenta fora do seu próprio ambiente (Figura 7). A serapilheira da FOM é mais rica em nutrientes e compostos orgânicos disponíveis, devido à maior diversidade de espécies arbóreas, disponibilizando, portanto, recursos mais diversificados para a comunidade decompositora (SCHMIDT et al., 2008). Ao contrário, as monoculturas apresentam recursos limitados e pouco diversificados quando comparadas a florestas nativas ou plantios consorciados (BLAIR et al., 1990; COÛTEAUX et al., 1995; CHAPMAN; NEWMAN, 2010).

\section{CONCLUSÕES}

A respiração microbiana e os atributos pedológicos diferem entre as três áreas, o que demonstra que a respiração microbiana é indicadora da cobertura florestal e que as características químicas do solo são influenciadas pela composição arbórea.

A comunidade de decompositores exerce papel significativo na decomposição da fitomassa. 
Existe uma elevada afinidade da macro e da mesofauna para decompor a serapilheira no seu local de origem, o que demonstra o importante papel desses organismos na decomposição da fitomassa em regiões tropicais.

\section{REFERÊNCIAS}

ASSIS JÚNIOR, S. L.; ZANUNCIO, J. C.; KASUYA, M. C. M.; COUTO, L.; MELIDO, R. C. N. Atividade microbiana do solo em sistemas agroflorestais, monoculturas, mata natural e área desmatada. Revista Árvore, Viçosa, v. 27, n. 1, p. 35-41, 2003. DOI: http://dx.doi.org/10.1590/S0100-67622003000100005

AYRES, E.; DROMPH, K. M.; BARDGETT, R. D. Do plant species encourage soil biota that specialize in the rapid decomposition of their litter? Soil Biology and Biochemistry, Elmsford, v. 38, n. 1, p. 183-186, 2006. DOI: https://doi.org/10.1016/j.soilbio.2005.04.018

BERNARDINO, F. S.; GARCIA, R. Sistemas silvipastoris. Pesquisa Florestal Brasileira, Colombo, n. 60, p. 7787, 2009. DOI: https://doi.org/10.4336/2009.pfb.60.77

BLAIR, J. M.; PARMELEE, R. W.; BEARE, M. H. Decay rates nitrogen fluxes and decomposer communities of single and mixed species foliar litter. Ecology, Brooklyn, v. 71, n. 5 , p. 1976-1985, 1990. DOI: https://doi.org/10.2307/1937606

CARDOSO, F. C. G.; MARQUES, R.; BOTOSSO, P. C.; MARQUES, M. C. M. Stem growth and phenology of two tropical trees in contrasting soil conditions. Plant and Soil, The Hague, v. 354, p. 269-281, 2012. DOI: https://doi.org/10.1007/s11104-011-1063-9

CARVALHO, J. G. (Ed.). Inter-relação fertilidade, biologia do solo e nutrição de plantas. Viçosa: SBCS, Lavras: UFLA/DCS, 1999. 818p.

CASTElla, P. R.; BRITEZ, R. M. A Floresta com Araucária no Paraná: conservação e diagnóstico dos remanescentes florestais. Brasília: Fundação de Pesquisas Florestais do Paraná, 2004. 233p.

CHAPMAN, S. K.; NEWMAN, G. S. Biodiversity at the plant-soil interface: microbial abundance and community structure respond to litter mixing. Oecologia, Berlin, v. 162, n. 3, p. 763-769, 2010. DOI: https://doi.org/10.1007/s00442-009-1498-3

COUTEAUX, M.; BOTTNER, P.; BERG, B. Litter decomposition, climate and litter quality. Trends in Ecology and Evolution, Amsterdam, v. 10, n. 2, p. 6366, 1995. DOI: https://doi.org/10.1016/S01695347(00)88978-8

FANIN N.; FROMIN N.; BERTRAND I. Functional breadth and home-field advantage generate functional differences among soil microbial decomposers. Ecology, Brooklyn, v. 97, p. 1023-1037, 2016. DOI: https://doi.org/10.1890/15-1263.1

FANIN, N.; BEZAUD, S.; SARNEEL, J. M.; CECCHINI, S.; MANUEL N.; LAURENT, A. Relative importance of climate, soil and plant functional traits during the early decomposition stage of standardized litter. Ecosystems, New York, v. 23, p. 1004-1018, 2019. DOI: https://doi.org/10.1007/s10021-019-00452-z

GARCIA-PALACIOS, P.; MAESTRE, F. T.; KATTGE, J.; WALL, D. H. Climate and litter quality differently modulate the effects of soil fauna on litter decomposition across biomes. Ecology Letters, Oxford, v. 16, p. 10451053, 2013. DOI: https://doi.org/10.1111/ele.12137
GIACOMETTI, C.; DEMYAN, M. S.; CAVANI, L.; MARZADORI, C.; CIAVATTA, C.; KANDELER, E. Chemical and microbiological soil quality indicators and their potential to differentiate fertilization regimes in temperate agroecosystems. Applied Soil Ecology, Amsterdam, v. 64 , p. 32-48, 2013. DOI: https://doi.org/10.1016/j.apsoil.2012.10.002

GIEßELMANN, U. C.; MARTINS, K. G.; BRÄNDLE, M.; SCHÄDLER, M.; MARQUES, R. BRANDL, R. Diversity and ecosystem functioning: litter decomposition dynamics in the Atlantic Rainforest. Applied Soil Ecology, Amsterdam, v. 46, n. 2, p.283290, 2010. https://doi.org/10.1016/j.apsoil.2010.07.006

GIEßELMANN, U. C.; MARTINS, K. G.; BRÄNDLE, M.; SCHÄDLER, M.; MARQUES, R.; BRANDL, R. Lack of home-field advantage in the decomposition of leaf litter in the Atlantic Rainforest of Brazil. Applied Soil Ecology, Amsterdam, v. 49, p. 5-10, 2011. https://doi.org/10.1016/j.apsoil.2011.07.010

GHOLZ, H. L.; WEDIN, D. A.; SMITHERMAN, S. M.; HARMON, M. E.; PARTON, W. J. Long-term dynamics of pine and hardwood litter in contrasting environments: toward a global model of decomposition. Global Change Biology, Oxford, v. 6, n. 7, p. 751-765, 2000. https://doi.org/10.1046/j.1365-2486.2000.00349.x

GUZMÁN, G. B.; SÁNCHEZ, J. A. The relationships between litter fauna and rates of litter decomposition in a tropical rain forest. Applied Soil Ecology, Amsterdam, v. 24 , n. 1, p. 91-100, 2003. DOI: https://doi.org/10.1016/S0929-1393(03)00069-6

HENDRIX, P. F.; CROSLEY JR, D. A.; BLAIR, J. M.; COLEMAN, D. C. Soil biota as component of sustainable agroecosystems. In: EDWARDS, C. A.; LAL, R.; MADDEN, P.; MILLER, R. H.; HOUSE, G. (Eds.). Sustainable Agricultural Systems. Ankey: Soil and Water Conservation Society, 1990. p. 637-654.

HENEGHAN, L; COLEMAN, D. C; ZOU, X.; CROSSLEY JR, D. A.; HAINES, B. L. Soil microarthropod community structure and litter decomposition dynamics: a study of tropical and temperate sites. Soil Ecology, v. 9, n. 1, p. 33-38, 1998. DOI: https://doi.org/10.1016/S0929-1393(98)00050-X

HOOPER, E. R.; LEGENDRE, P.; CONDIT, R. Factors affecting community composition of forest regeneration in deforested, abandoned land in Panama. Ecology, Brooklyn, v. 85 , n. 12 , p. 3313-3326, 2004. DOI: https://doi.org/10.1890/03-0655

JENKINSON, D. S.; POWLSON, D. S. The effects of biocidal treatments on metabolism in soil - V. A method for measuring soil biomass. Soil Biology and Biochemistry, Oxford, v. 8, n. 3, p. 209-213, 1976. DOI: https://doi.org/10.1016/0038-0717(76)90005-5

LAVELLE, P.; BLANCHART, E.; MARTIN, A.; MARTIN, S.; SPAIN, A. A hierarchical model for decomposition in terrestrial ecosystems: application to soils of the humid tropics. Biotropica, Hoboken, v. 25, n. 2, p. 130-150, 1993. https://doi.org/10.2307/2389178

MAGURRAN, A. E. Measuring Biological Diversity. Maldem: Blackwell Publishing, 2011. 256p.

MASON, C. F. Decomposição. São Paulo: Ed. da Universidade de São Paulo, 1980. 63p.

MASON, N. W. H.; CARSWELL, F. E.; RICHARDSON, S. J.; BURROWS, L. E. Leaf palatability and decomposability increase during a 200-year-old post 
cultural woody succession in New Zealand. Journal of Vegetation Science, Knivsta, v. 22, n. 1, p. 6-17, 2011. DOI: https://doi.org/10.1111/j.16541103.2010.01223.x

McCUNE, B.; GRACE, J. B. Analysis of Ecological Communities. Oregon: MJM, 2002. 300p.

MILLENNIUM ECOSYSTEM ASSESSMENT (MEA). Ecosystems and human well-being: synthesis. Washington: Island Press, 2005. 156 p.

MOREIRA, F. M. S.; SIQUEIRA, J. O. Microbiologia e Bioquímica do Solo. Lavras: Editora UFLA, 2002. 626p.

OSTERTAG, R.; MARÍN-SPIOTTA, E.; SILVER, W. L.; SCHULTEN, J. Litterfall and decomposition in relation to soil carbon pools along a secondary forest chronosequence in Puerto Rico. Ecosystems, New York, v. 11, n. 5, p. 701-714, 2008. DOI: https://doi.org/10.1007/s10021-008-9152-1

SCHMIDT, P.; DICKOW, K.; ROCHA, A.; MARQUES, R.; SCHEUERMANN, L.; RÖMBKE, J.; FÖRSTER, B.; HÖFER, H. Soil macrofauna and decomposition rates in southern Brazilian Atlantic rainforests. Ecotropica, Bonn, v. 14, p. 89-100, 2008.

SMYTH, C. E.; KURZ, W. A.; TROFYMOW, J. A. Including the effects of water stress on decomposition in the carbon budget. Model of the Canadian forest sector. Ecological Modelling, Amsterdam, v. 222, n. 5, p. 1080-1091, 2011.2 DOI: https://doi.org/10.1016/j.ecolmodel.2010.12.005

TOWNSEND, A. R.; ASNER, G. P.; CLEVELAND, C. C. The biogeochemical heterogeneity of tropical forests. Trends in Ecology \& Evolution, Amsterdam, v. 23, n. 8, p. 424-431, 2008. DOI: https://doi.org/10.1016/j.tree.2008.04.009

TOWNSEND, C. R.; BEGON, M.; HARPER, J. L. Fundamentos em ecologia. São Paulo: Editora Artmed, 2010. 576p.

VARGAS, L. K.; SCHOLLES, D. Biomassa microbiana e produção de $\mathrm{C}-\mathrm{CO}_{2}$ e $\mathrm{N}$ mineral de um Podzólico Vermelho-Escuro submetido a diferentes sistemas de manejo. Revista Brasileira de Ciência do Solo, Viçosa, v. 24 , n. 1 , p. 35-42, 2000. DOI: https://doi.org/10.1590/S0100-06832000000100005

ZAR, J. H. Biostatistical analysis. New Jersey: PrenticeHall, 1999. 666p.

ZILLI, J. E.; RUMJANEK, N. G.; XAVIER, G. R.; COUTINHO, H. L. C.; NEVES, M. C. P. Diversidade microbiana como indicador de qualidade do solo. Cadernos de Ciência \& Tecnologia, Brasília, v. 20, n. 3, p. 391-411, 2003. DOI: http:/ /dx.doi.org/10.35977/01041096.cct2003.v20.8751 